\title{
Constraints on multiparticle production in scalar field the- ory from classical simulations
}

\author{
S.V. Demidov ${ }^{1,2, *}$ and B.R. Farkhtdinov ${ }^{1,2, * *}$ \\ ${ }^{1}$ Institute for Nuclear Research of the Russian Academy of Sciences, \\ 60th October Anniversary prospect 7a, Moscow 117312, Russia \\ ${ }^{2}$ Moscow Institute of Physics and Technology, \\ Institutsky per. 9, Dolgoprudny 141700, Russia
}

Abstract. We report numerical results on classical scattering of waves in a scalar field theory and discuss their connection to multiparticle production.

\section{Introduction}

We start with some known results on multiparticle production in standard $\lambda \phi^{4}$ scalar field theory in 3+1 dimensions. Tree level calculations [1-4] show that the amplitudes of multiparticle production, e.g. $1 \rightarrow N$ process, grow factorially at the threshold with number $N$ of final particles. Corresponding probability also shows factorial growth and it can be written as follows $(\lambda \ll 1$ is assumed $)$

$$
\mathcal{P}_{1 \rightarrow N}^{\text {tree }} \sim N ! \lambda^{N} \mathrm{e}^{N f(\mathcal{E})} \sim \exp \left(\frac{1}{\lambda} F(\lambda N, \mathcal{E})\right), \quad \mathcal{E}=\frac{E-N m}{N},
$$

where $E$ is total energy of colliding particles and $m$ is mass of field quanta. Inspired by this exponential form, semiclassical methods to calculate the probability were developed [57] which among other things allowed to estimate its energy dependence. In particular, at ultrarelativistic regime the probability is energy independent and its behaviour as function of $\lambda N$ is shown in Figure 1. One can see that it is exponentially suppresses up to $\lambda N$ of order 200. Let us note that corresponding calculations are valid only at $\lambda N \ll 1$ while at larger $\lambda N$ loop corrections are important. Also there are unitary-base arguments $[9,10]$ which tell that this probability should be exponentially suppressed even at large $\lambda N$ and energies $E$ but actual behavior of the multiparticle probability in this regime is still unknown.

Let us make several steps away from the problem of calculation of multiparticle amplitudes. Firstly, let us consider instead of $f e w \rightarrow N$ processes the scattering processes in which both initial and final particle numbers are large, i.e. $N_{i} \rightarrow N_{f}, N_{i}, N_{f} \sim 1 / \lambda$. In this case both initial and final states of collision process are semiclassical and it is natural to study this system from the semiclassical point of view. Next, if these particle numbers are large it is natural to study classical solutions to the field equations, which describe scattering of waves. Namely, if we consider solutions which linearize at infinite times corresponding initial and final states can be associated with coherent states with some average particle numbers $N_{i}$,

\footnotetext{
*e-mail: demidov@ms2.inr.ac.ru

**e-mail: farkhtdinov@phystech.edu
} 


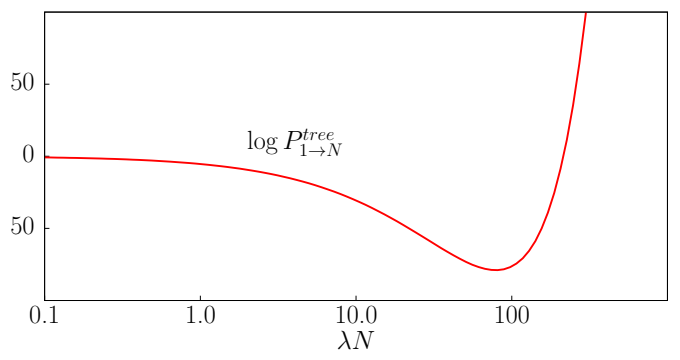

Figure 1. Particle number dependence of $\log P_{1 \rightarrow N}^{\text {tree }}$, see e.g. [8].

$N_{f}$ and energy $E$. Common lore here is that if such a classical solution with some particular particle numbers $N_{i}, N_{f}$ and energy $E$ exists then corresponding quantum scattering process is classically allowed and thus its probability is not exponentially suppressed. In the opposite case such a process belongs to classically forbidden region and its probability is expected to be suppressed exponentially. In this talk we report numerical results [11] on classical scattering of wave packets in $\lambda \phi^{4}$ theory.

\section{The method}

For convenience we rescale coordinates and field to dimensionless units as $x \rightarrow m^{-1} x$ and $\phi \rightarrow \sqrt{\frac{m^{2}}{\lambda}} \phi$ and obtain action in the form

$$
S[\phi]=\frac{1}{\lambda} \int d^{4} x\left[\frac{1}{2}\left(\partial_{\mu} \phi\right)^{2}-\frac{1}{2} \phi^{2}-\frac{1}{4} \phi^{4}\right] .
$$

After that the equation of motion becomes independent of coupling constant which appears as a factor in front of the action and plays a role of semiclassical parameter. In what follows we limit ourselves to spherically symmetric field configurations and in this case it is useful to substitute following anzatz $\phi(t, r)=\frac{1}{r} \chi(t, r)$ and obtain the action in the form

$$
S=\frac{4 \pi}{\lambda} \int d t d r\left[\frac{1}{2}\left(\frac{\partial \chi}{\partial t}\right)^{2}-\frac{1}{2}\left(\frac{\partial \chi}{\partial r}\right)^{2}-\frac{\chi^{2}}{2}-\frac{\chi^{4}}{4 r^{2}}\right] .
$$

In what follows we solve classical e.o.m. numerically. To do this we restrict our solutions to a finite space interval $r \in[0, R]$ where $R$ is sufficiently large. At boundaries we impose conditions $\chi(t, r=0)=0$ and $\partial_{r} \chi(t, r=R)=0$. The boundary conditions on the field $\chi$ allow us to expand field configuration as follows

$$
\chi(t, r)=\sum_{n=0}^{\infty} c_{n}(t) \sqrt{\frac{2}{R}} \sin k_{n} r,
$$

where $k_{n}$ are corresponding wave numbers. We study solutions which linearize at initial and final times. In the linear regime the time-dependent Fourier components $c_{n}(t)$ can be conveniently written via positive and negative frequency components as follows

$$
c_{n}(t) \rightarrow \begin{cases}\frac{1}{\sqrt{2 \omega_{n}}}\left(a_{n} \mathrm{e}^{-i \omega_{n} t}+a_{n}^{*} \mathrm{e}^{i \omega_{n} t}\right) & \text { as } t \rightarrow-\infty \\ \frac{1}{\sqrt{2 \omega_{n}}}\left(b_{n} \mathrm{e}^{-i \omega_{n} t}+b_{n}^{*} \mathrm{e}^{i \omega_{n} t}\right) & \text { as } t \rightarrow+\infty\end{cases}
$$


Using these representations one can compute the energy of colliding wave packets as well as initial and final particle numbers

$$
E=\frac{4 \pi}{\lambda} \sum_{n} \omega_{n}\left|a_{n}\right|^{2}=\frac{4 \pi}{\lambda} \sum_{n} \omega_{n}\left|b_{n}\right|^{2}, \quad N_{i}=\frac{4 \pi}{\lambda} \sum_{n}\left|a_{n}\right|^{2}, \quad N_{f}=\frac{4 \pi}{\lambda} \sum_{n}\left|b_{n}\right|^{2},
$$

where $\left.\omega_{n}=\sqrt{(} k_{n}^{2}+1\right)$. For convenience we introduce the notations $\tilde{E}=\frac{\lambda}{4 \pi} E, \quad \tilde{N}_{i}=$ $\frac{\lambda}{4 \pi} N_{i}, \quad \tilde{N}_{f}=\frac{\lambda}{4 \pi} N_{f}$. For numerical implementation of the field equations on $c_{n}(t)$ we truncate the Fourier expansion (3) at finite number of terms $n=N_{r}$ and solve evolution equations using Bulirsch-Stoer method.

We take initial conditions which correspond to a wave packet which is localized well away from the interaction region and which propagates towards $r=0$. We choose an interval $I=\left[r_{1}, r_{2}\right]$ and construct initial wave packet as following superposition

$$
\chi(r)=\left\{\begin{array}{l}
\sum f_{n} \sin \left(\tilde{k}_{n}\left(r-r_{1}\right)\right), r \in I \\
0, r \notin I
\end{array}\right.
$$

wave numbers $\tilde{k}_{n}$ are chosen in such a way that this wave packet nullifies at the boundaries of the interval $I$. The first derivative of the field at initial time $t_{i n}$ is chosen in such a way that the wave packet starts propagating to the left:

$$
\chi_{i}(t, r)=\left\{\begin{array}{l}
\sum f_{n} \sin \left(\tilde{k}_{n}\left(r-r_{1}\right)+\tilde{\omega}_{n}\left(t-t_{i n}\right)\right), \quad r \in\left[r_{1}, r_{2}\right] \\
0, r \notin\left[r_{1}, r_{2}\right] .
\end{array}\right.
$$

Schematic view of the initial wave packet is shown in Fig. 2.

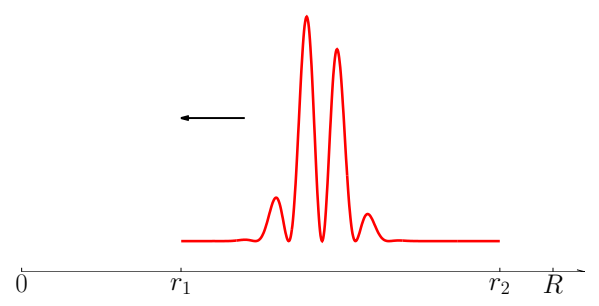

Figure 2. Schematic view of initial wave packet.

Our aim is to find classical solutions describing scattering of wave packets in which particle number changes as much as possible at a given energy. This corresponds to finding absolute minimum (or maximum) of $\tilde{N}_{f}$ at fixed energy and initial particle number with respect to initial conditions, i.e. Fourier amplitudes $f_{n}$. To find its absolute minimum (maximum) we use stochastic sampling technique in combination with simulated annealing method, see e.g. [12, 13]. Namely, we generate an ensemble of the classical solutions with fixed initial particle number $\tilde{N}_{i}$ weighted by the following probability $p \sim \mathrm{e}^{-F}$, where $F=\beta\left(\tilde{N}_{f}+\xi\left(\tilde{E}-\tilde{E}_{*}\right)^{2}\right)$. At large positive $\beta$ and $\xi$ the ensemble will be dominated by solutions having small $F$ thus they will concentrate near the smallest possible values of $\tilde{N}_{f}$ at energy $\tilde{E}_{*}$. To reach the the upper boundary $\tilde{N}_{f}$ at energy $E_{*}$ the signs of $\beta$ and $\xi$ should be negative. To generate such ensemble we use Metropolis Monte Carlo 
algorithm and vary values of $\beta$ and $\xi$ to reach the boundary of classically allowed solution in the parameter space $\left(\tilde{E}, \tilde{N}_{f}\right)$, see Ref. [11] for details. In the next Section we present obtained results.

\section{Results}

Below we show regions of the classical solutions in the parameter space $\left(\tilde{E}, \tilde{N}_{f}\right)$ for several different values of initial particle number $\tilde{N}_{i}$. In Fig. 3 we plot the results of the stochastic

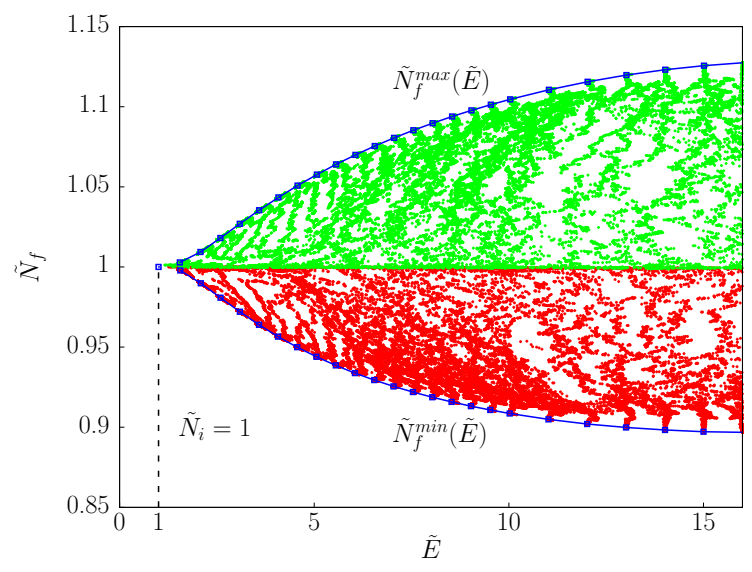

Figure 3. Classically allowed region for wave packet scattering process in $\left(\tilde{E}, \tilde{N}_{f}\right)$ plane.

sampling in the plane $\left(\tilde{E}, \tilde{N}_{f}\right)$ for $\tilde{N}_{i}=1$. Here we fix $R=20$ and $N_{r}=400$. Each dot in the Fig. 3 represents a classical solution obtained on the way to the lower and upper boundaries. The picture is a combination of several runs with several different values of $E_{*}$ chosen to populate representative energy interval. The very first solution of our procedure was randomly chosen with the only conditions that it is linear at initial and final times and $\tilde{N}_{i}=1$. Such initial solutions typically have very small change in particle number during the time evolution and thus are situated near the horizontal line $\tilde{N}_{f}=1$ on this figure. Then we run our procedure as described above. The obtained region of the classical solutions in this plane has a smooth envelopes, $\tilde{N}_{f}^{\min }(\tilde{E})$ and $\tilde{N}_{f}^{\max }(\tilde{E})$ which represent boundary of the classically allowed region in the parameter space.

In Fig.4 we show typical time evolution of an exemplary upper boundary solution with $\tilde{E} \approx 6.0$. Form of the initial wave packet is the same for all boundary solutions which we have found for $\tilde{N}_{i}=1$. Namely, it has relatively sharp part near its end where most of its energy is concentrated. The wave packet reaches interaction region, reflects and goes back to the linear regime and the final wave packet has similar form as the initial one.

We study the dependence of the obtained results on the lattice, namely on the spacial cutoff $R$ and on number of Fourier modes $N_{r}$. We repeat the same numerical procedure to find the lower and upper boundaries, for the case $R=20, N_{x}=600$ with smaller lattice spacing and for the case $R=30, N_{x}=600$ with larger space interval but the same lattice spacing. We have found nice coincidence in the position of the boundaries of classically allowed region and form of corresponding solution. A deviation has been observed for the case $N_{x}=600, R=20$ for energies larger than 10 which reflects appearance of new high energy modes. 


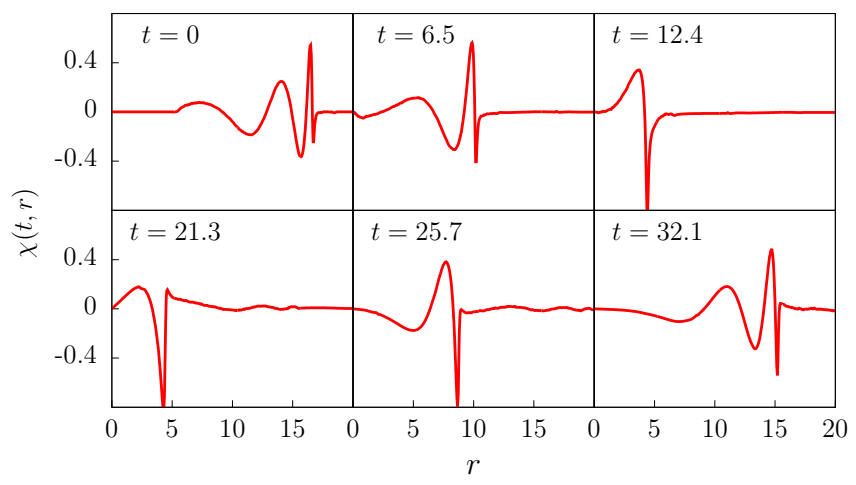

Figure 4. Time evolution of an exemplary upper boundary solution at $\tilde{E} \approx 6.0$.

Now we turn to discussion of numerical results for another values of initial particle number. In Fig. 5 the boundary of the classically allowed region for $\tilde{N}_{i}=10$ is shown with thick

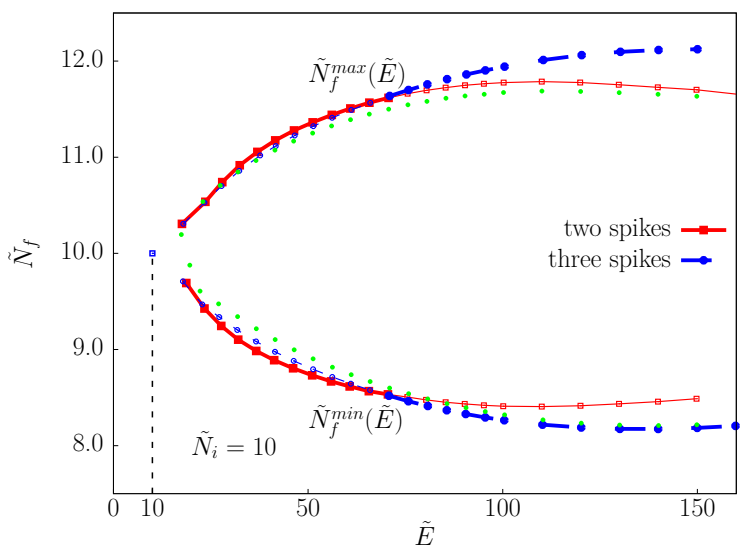

Figure 5. Boundaries of classically allowed region for $\tilde{N}_{i}=10$.

solid (red) and thick dashed (blue) lines. We find that although the form of the boundary is in the whole similar to that of for smaller values of $N_{i}$, its upper and lower parts actually consist of two different branches of classical solutions. At energies lower than about 70 the boundary solutions have two distinct spiky parts in its initial wave packet. Corresponding example is presented in the left panel of Fig. 6. We observe that the configuration consists of two similar but space shifted configurations. At energies larger than about 70 the boundary solutions have three spikes in the initial and final wave packet. Example of such a solution is presented in Fig. 6, right panel. In this branch of solutions the initial and final field configurations looks as a sum of three separated in space wavetrains with similar Fourier image. Upper boundary solutions have very similar properties.

The picture becomes even more complicated at larger values of $N_{i}$. For $\tilde{N}_{i}=30$ we have found that the corresponding boundary solutions contain already 4-7 spikes in the initial (and 

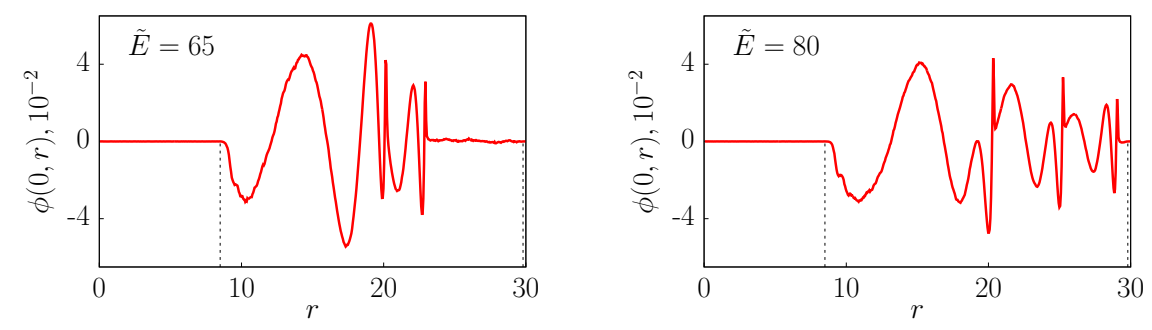

Figure 6. Initial wave packets of lower boundary solutions for $\tilde{E} \approx 65$ (left panel) and $\tilde{E} \approx 80$ (right panel).

final) wavetrains. We note that the task of finding the boundaries becomes more complicated in this case due to the presence of multiple local extrema of $N_{f}$. They correspond to solutions with larger distances between spikes in the initial wave packets. Our results indicate that at larger $N_{i}$ the initial wave packets of boundary solutions tend to consist of more and more space separated wavetrains.

It is easy to see that if two classical boundary solutions with different sets of parameters exist then there should exist also a classical solution describing scattering which has energy and particle numbers equal to the sums of the corresponding energies and particle numbers of these two solutions. One can construct this solution explicitly by taking it as a sufficiently separated in space sum of those two solutions. In particular, this means that the width of the classically allowed region in parameter space $\left(\tilde{E}, \tilde{N}_{f}\right)$ should grow with increase of initial particle number faster than linear function. Comparison of classically allowed regions for different values of $\tilde{N}_{i}$ is shown in Fig. 7 (left panel). On the right panel of the Figure we
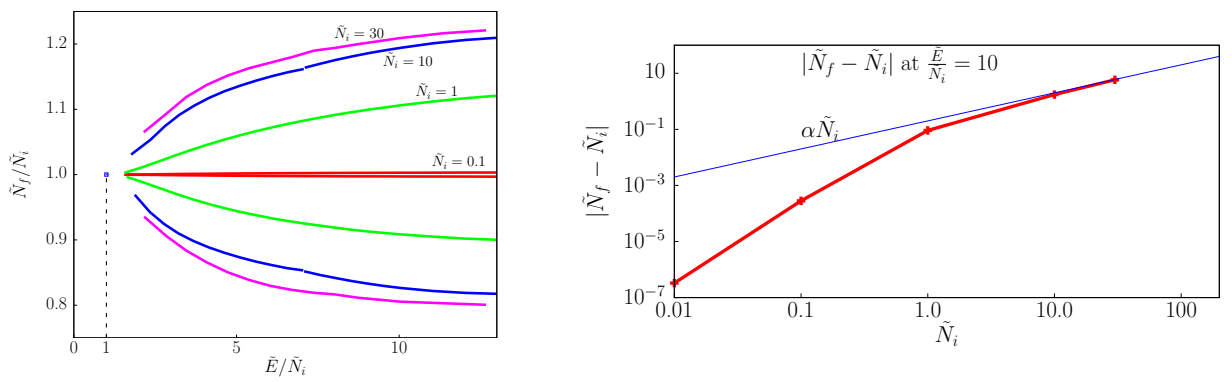

Figure 7. Left panel: comparison of classically allowed regions for different values of $\tilde{N}_{i}$. Right panel: width of classically allowed region as a function of $\tilde{E} / \tilde{N}_{i}$.

present dependence of the difference of initial and final particle number for particular ratio $\tilde{E} / \tilde{N}_{i}=10$ as a function of initial particle number $\tilde{N}_{i}$. One can see that at large values of initial particle number the behaviour of this function tends to linear. This can be considered as an indication on existence of a limiting boundary of classically allowed region at large $N_{i}$.

Finally let us make a connection of our results with the most interesting case which is $2 \rightarrow N$ particle scattering where $N$ is semiclassically large, i.e. $\sim 1 / \lambda$. First, let us consider 
the following inclusive probability

$$
\mathcal{P}\left(N_{i}, N_{f}, E\right)=\sum_{i, f}\left|\left\langle i\left|\hat{P}_{N_{i}} \hat{S} \hat{P}_{N_{f}} \hat{P}_{E}\right| f\right\rangle\right|^{2},
$$

where $\hat{S}$ is $S$-matrix, $\hat{P}_{E}, \hat{P}_{N_{i}}$ and $\hat{P}_{N_{f}}$ are projectors onto subspaces with fixed energy, initial and final particle numbers and sum goes over all initial $i$ and final $f$ states. This quantity can be viewed as a total probability of transition from states with initial particle number $N_{i}$ and final particle number $N_{f}$ with energy $E$. Suppose that the process $N_{i} \rightarrow N_{f}$ scattering at energy $E$ consists of two subprocesses with some energies $E^{(1)}, E^{(2)}$ and particle numbers $N_{i}^{(1)}, N_{i}^{(2)}, N_{f}^{(1)}, N_{f}^{(2)}$. If these subprocesses are separated by very large distance then their total probability is given by the product $\mathcal{P}\left(E^{(1)}, N_{i}^{(1)}, N_{f}^{(1)}\right) \mathcal{P}\left(E^{(2)}, N_{i}^{(2)}, N_{f}^{(2)}\right)$. As such subprocesses represent a subset among all possible $N_{i} \rightarrow N_{f}$ scatterings at energy $E$ then the following inequality should be valid

$$
\mathcal{P}\left(N_{i}^{(1)}+N_{i}^{(2)}, N_{f}^{(1)}+N_{f}^{(2)}, E^{(1)}+E^{(2)}\right) \geq \mathcal{P}\left(N_{i}^{(1)}, N_{f}^{(1)}, E^{(1)}\right) \mathcal{P}\left(N_{i}^{(2)}, N_{f}^{(2)}, E^{(2)}\right) .
$$

Let us apply this property to subprocesses $2 \rightarrow N$ at energy $E$ and $N \rightarrow N$ at energy $E_{0}$, where $N$ is semiclassically large, i.e. $N_{f} \gtrsim 1 / \lambda$. We obtain following inequality

$$
\mathcal{P}\left(2+N, 2 N, E+E_{0}\right) \geq \mathcal{P}(2, N, E) \mathcal{P}\left(N, N, E_{0}\right)
$$

The process $N \rightarrow N$ scattering is classically allowed at all energies above the threshold. At the same time the initial state with $N+2$ number of particles is semiclassically indistinguishable from the state with particle number equal to $N$. And our numerical results indicate that processes $N \rightarrow 2 N$ are classically forbidden at least for considered range of energies. Then the above inequality tells us that the processes $2 \rightarrow N$ should be exponentially suppressed. Another even simpler argument in favor of suppression of $2 \rightarrow N_{f}$ processes follows from $T$-invariance which in particular ensures that $\mathcal{P}\left(E, N_{i}, N_{f}\right)=\mathcal{P}\left(E, N_{f}, N_{i}\right)$. The processes $N_{f} \rightarrow$ few correspond to the lowest part of the $\left(\tilde{E}, \tilde{N}_{f}\right)$ plane in the Figures 3 and 5 which implies that they lie deep in the classically forbidden region. This means that the probability of the process $N_{f} \rightarrow$ few and thus of the process $f e w \rightarrow N_{f}$ is expected to be exponentially suppressed.

\section{Conclusions}

In summary, we numerically study classically allowed region in the parameter space $\tilde{E}, \tilde{N}_{i}$, $\tilde{N}_{f}$ for processes describing scattering of waves in unbroken scalar $\lambda \phi^{4}$ theory and study properties of boundary solutions at different values of $\tilde{N}_{i}$. Our results indicate on existence of limiting (at $\tilde{N}_{i} \rightarrow \infty$ ) boundary region of classically allowed transitions and they imply that the probability of of $2 \rightarrow N$ processes at any $N$ (not only at small $\lambda N$ ) is exponentially suppressed. Of course, performed classical simulations tell nothing about actual value of probability of these processes. We plan to calculate suppression exponent semiclassically in the classically forbidden region starting with processes when both initial and final particle numbers are semiclassically large. In this case the problem is reduced to solution of a corresponding semiclassical boundary value problem and at the end by taking limit $\tilde{N}_{i} \rightarrow 0$, see Refs. [14-17]. When approaching the boundary of the classically allowed region the suppression exponent should go to zero which can be used as a check of our procedure.

\section{Acknowledgments}

The work was supported by the RSCF grant 14-22-00161. The numerical part of the work was performed on Calculational Cluster of the Theory Division of INR RAS. 


\section{References}

[1] J. M. Cornwall, Phys. Lett. B 243271 (1990) .

[2] H. Goldberg, Phys. Lett. B 246445 (1990).

[3] L. S. Brown, Phys. Rev. D 46 R4125 (1992) [hep-ph/9209203].

[4] M. B. Voloshin, Nucl. Phys. B 383233 (1992).

[5] S. Y. Khlebnikov, V. A. Rubakov and P. G. Tinyakov, Nucl. Phys. B 350441 (1991).

[6] V. A. Rubakov and P. G. Tinyakov, Phys. Lett. B 279165 (1992).

[7] D. T. Son, Nucl. Phys. B 477378 (1996) [hep-ph/9505338].

[8] F. L. Bezrukov, M. V. Libanov and S. V. Troitsky, Mod. Phys. Lett. A 102135 (1995) [hep-ph/9508220].

[9] V. I. Zakharov, Phys. Rev. Lett. 673650 (1991).

[10] V. A. Rubakov, hep-ph/9511236.

[11] S. V. Demidov and B. R. Farkhtdinov, arXiv:1806.10996 [hep-ph].

[12] C. Rebbi and R. L. Singleton, Jr, Phys. Rev. D 541020 (1996) [hep-ph/9601260].

[13] S. V. Demidov and D. G. Levkov, JHEP 1106016 (2011) [arXiv:1103.2133 [hep-th]].

[14] F. L. Bezrukov, D. Levkov, C. Rebbi, V. A. Rubakov and P. Tinyakov, Phys. Rev. D 68 036005 (2003) [hep-ph/0304180].

[15] D. G. Levkov and S. M. Sibiryakov, Phys. Rev. D 71025001 (2005) [hep-th/0410198].

[16] S. V. Demidov and D. G. Levkov, Phys. Rev. Lett. 107071601 (2011) [arXiv:1103.0013 [hep-th]].

[17] S. V. Demidov and D. G. Levkov, JHEP 1511066 (2015) [arXiv:1509.07125 [hep-th]]. 